\title{
ACIDENTES DE TRABALHO COM CRIANÇAS E ADOLESCENTES: DADOS CONHECIDOS OU DADOS OCULTADOS?
}

\author{
Lorraine Lacerda Leite \\ Universidade Federal da Paraíba \\ Gabriela Fernandes Rocha \\ Universidade Federal da Paraíba \\ Maria de Fátima Pereira Alberto \\ Universidade Federal da Paraíba
}

\begin{abstract}
Resumo
Trabalho precoce é um problema de saúde pública, pois expõe crianças e adolescentes a riscos, lesões e deformidades físicas, afetando seu desenvolvimento. Este estudo teve o objetivo de caracterizar acidentes de trabalho que envolvem crianças e adolescentes do município de João Pessoa. Utilizou-se análise descritiva das informações de 62 fichas. Verificou-se que a maioria dos acidentes ocorreu com meninos $(95,7 \%)$, idade de 16 a 18 anos $(77,4 \%)$, exercendo atividades no setor de comércio e serviços $(46,4 \%)$, causados por contato com objetos cortantes $(37,1 \%)$, provocando ferimentos $(27,4 \%)$ e contusões $(22,6 \%)$ nos membros superiores $(56,5 \%)$. Constatou-se que as fichas são preenchidas de forma inadequada, com respostas em branco, não especificado ou ignorado. Concluiu-se que o monitoramento dos agravos e prejuízos à saúde provocados por acidentes de trabalho com crianças e adolescentes constitui uma forma de contribuir com ações nos diversos níveis da rede pública de saúde, voltadas para erradicação do trabalho precoce.

Palavras-chave: trabalho infantil; acidentes de trabalho; políticas públicas; saúde.
\end{abstract}

\section{WORK ACCIDENTS WITH CHILDREN AND ADOLESCENTS: KNOWN DATA OR HIDDEN DATA?}

\begin{abstract}
Child labor is a public health problem, because it exposes children and adolescents to risks, injuries and physical deformities, affecting their development. This study aimed to characterize the work accidents involving children and adolescents of the city of João Pessoa. It was used a descriptive analysis of the information contained in 62 cards. It was found that the majority accidents occurred in boys (95,7\%), 16 to 18 years of age $(77,4 \%)$, exercising activities in trade and services sector $(46,4 \%)$, caused by contact with sharp slashing objects $(37,1 \%)$, causing injury $(27,4 \%)$ and contusions $(22,6 \%)$ in the upper limbs $(56,5 \%)$. It was also found of items improperly filled in the cards, with blank, not specified or ignored answers. It was concluded that the monitoring of the injures and health damage caused by work accidents with children and adolescents is a way of contributing to actions at all levels of public health, aimed to eradicate child labor.

Keywords: child labor; work accidents; public politics; health.
\end{abstract}




\title{
ACCIDENTES DE TRABAJO CON NIÑOS E ADOLESCENTES: ¿DATOS O DATOS OCULTOS CONOCIDO?
}

\begin{abstract}
Resumen
El trabajo infantil es un problema de salud pública, ya que expone a niños/as y adolescentes a riesgos, lesiones y deformidades físicas, afectando su desarrollo. El objetivo de este estudio fue describir los accidentes de trabajo sufridos por niños/as y adolescentes en la ciudad de João Pessoa. Utilizo el análisis descriptivo de las informaciones de 62 fichas, donde fue encontrado que la mayoría de los accidentes se pasa con los varones $(95,7 \%)$, edades entre 16 y 18 años $(77,4 \%)$, en las actividades del sector de los servicios y comercio $(46,4 \%)$. Suelen ser causados por objetos puntiagudos $(37,1 \%)$, generando lesiones $(27,4 \%)$ y contusiones $(22,6 \%)$ en las extremidades superiores $(56,5 \%)$. Fueron encontradas fichas rellenadas de manera inadecuada, con informaciones no especificadas y a veces ignoradas. Se concluye que el monitoreo de los accidentes y daños causados a la salud de niños/as y adolescentes trabajadores es una forma de contribuir para las acciones en todos los niveles de la salud pública, con el objetivo de erradicar el trabajo infantil.
\end{abstract}

Palabras clave: trabajo infantil; accidentes de trabajo; políticas públicas; salud.

\section{INTRODUÇÃO}

O trabalho infantil é conceituado pela Organização Internacional do Trabalho (OIT) como situações de trabalho que privam as crianças de sua infância, potencial e dignidade, prejudicando seu desenvolvimento físico e psicológico (Organização Internacional do Trabalho, 2004). Segundo Alberto (2007), o termo "precoce" se adequa melhor ao conceito por passar a ideia de imaturidade, não se relacionando apenas à infância, mas também à adolescência. Desse modo, o trabalho precoce se refere às "atividades variadas, a cuja ação objetiva está inerente a intenção de obter pagamento, que pode ser em espécie ou em gênero" (p. 9).

A legislação brasileira proíbe trabalho realizado por menores de 16 anos, salvo na condição de aprendiz, a partir dos 14 anos. Entende-se como aprendiz aquele que faz parte de um programa de formação técnico-profissional de aprendizagem, obedecendo às diretrizes e bases da legislação em vigor. De modo semelhante, a legislação proíbe, até os 18 anos, o trabalho noturno, insalubre, perigoso ou penoso, realizado em local que prejudique a formação e o desenvolvimento físico, psíquico, moral e social do trabalhador (Presidência da República, 1988/2017). As atividades que oferecem mais riscos à saúde, à moral e ao desenvolvimento estão descritas de forma associada com os prováveis riscos e repercussões à saúde, na Lista de Piores Formas de Trabalho Infantil (Lista TIP). Dentre outros pode-se citar: agricultura, pesca, indústria de transformação, construção, reparação de serviços automotores e serviços domésticos (Presidência da República, 2008). De modo geral, este conjunto de leis, orientado pelos princípios da Constituição Federal de 1988, busca convergir com os propósitos da Convenção dos Direitos das Crianças, da Organização das Nações Unidas (ONU) e as convenções 138 e 182 da Organização Internacional do Trabalho (OIT) (Stropasolas, 2012). 
Mesmo considerando-se os limites legais impostos sobre a idade mínima de admissão ao emprego, segundo dados do Instituto Brasileiro de Geografia e Estatística (IBGE), em 2012, na semana de referência (que precede a semana de entrevista), havia 3,4 milhões de crianças e adolescentes com idades entre $10 \mathrm{e}$ 17 anos ocupados. Na Paraíba, em um universo de 69,5 mil crianças e adolescentes classificadas como ocupadas, 35,5 mil estão na zona urbana e 33,9 mil na zona rural; destas da zona urbana, 6.234 estão localizadas em João Pessoa, capital do estado (IBGE, 2013). De acordo com relatório do Sistema de Indicadores Municipais de Trabalho Decente publicado pela Organização Internacional do Trabalho (OIT), considerando-se a faixa de 10 a 13 anos, na qual o trabalho precoce é terminantemente proibido, o município contava com 1.178 crianças desenvolvendo atividades laborais em situação irregular. Com idades entre 14 ou 15 anos, 1.389 crianças e adolescentes estavam trabalhando, das quais $97,7 \%$ estavam em situação irregular, ou seja, não contratados como aprendizes. Em relação a adolescentes de 16 ou 17 anos, 3.667 adolescentes estavam inseridos no mercado de trabalho (OIT, 2014).

Dados do Suplemento Especial denominado "Aspectos Complementares de Educação, Afazeres Domésticos e Trabalho Infantil" da Pesquisa Nacional por Amostra de Domicílio (PNAD), realizado pelo IBGE, revelam que das 5,1 milhões de crianças e adolescentes em situação de trabalho na semana de referência, 273 mil tiveram algum machucado ou doença em decorrência do trabalho. Ainda segundo o Suplemento Especial, a região em que crianças e adolescentes de 5 a 17 anos sofreram mais machucados ou doenças devido ao trabalho, em relação a todas as crianças ocupadas na semana de referência, foi a Nordeste $(7,1 \%)$. Esse valor está acima da média nacional que é 5,3\% (IBGE,2008).

De acordo com o Plano Nacional de Prevenção e Erradicação do Trabalho Infantil e Proteção do Adolescente Trabalhador (Comissão Nacional de Erradicação do Trabalho Infantil, 2011), o Brasil teceu como meta erradicar as piores formas de trabalho precoce até 2016 e, até 2020, eliminar todas as suas formas. Para tal, o combate ao trabalho precoce é planejado a partir de funções e papéis em diferentes níveis estatais, a fim de garantir direitos através de políticas públicas intersetoriais, como assistência social, formação profissional, educação e saúde.

É importante compreender o problema do trabalho precoce em seu contexto macrossocial: "o modo de produção capitalista se aproveita da condição de precariedade das famílias, ao mesmo tempo que a cria e a reproduz" (Lourenço, 2014, p. 300). Para Stropasolas (2012), ações para eliminar o trabalho precoce serão infrutíferas se não abrangerem os efeitos perversos do mercado de trabalho, como: baixos salários, controle e dependência dos trabalhadores aos patrões. Segundo Alberto (2002), a mão de obra infantil contemporânea se dá principalmente na economia informal. O termo "setor informal" diz respeito a ocupações como serviços domésticos, agricultura, vendas 
ambulantes e uma série de atividades de fabricação e serviços. As atividades realizadas nesse setor são precárias e não oferecem segurança no trabalho (OIT, 2004). Esta informalidade do trabalho realizado por crianças e adolescentes é o maior obstáculo para a erradicação do trabalho precoce (Lourenço, 2014).

O trabalho precoce é naturalizado na nossa sociedade. Estabeleceu-se um consenso social que crianças que pertencem às classes menos favorecidas devem ser inseridas no mercado de trabalho, com a justificativa de prevenção à marginalidade e formação de caráter, mesmo que ocorra em situação precária e insegura, apresentando riscos à saúde da pessoa em condição peculiar de desenvolvimento (Lourenço, 2014).

As principais preocupações em relação à saúde de crianças e adolescentes inseridas no mercado de trabalho são o risco e a vulnerabilidade aos quais estão expostos, pois doenças e lesões são comuns em situação de trabalho devido ao sistema biológico não estar maduro até os 18 anos de idade associado à pouca coordenação motora (Lacorte, 2012). Dados revelam que o trabalho precoce acarreta danos à saúde e ao desenvolvimento das crianças e adolescentes, pois estes estão mais vulneráveis do que adultos aos riscos proporcionados pelo trabalho (Giatti, Campo, Crespo, Andrade, \& Barreto, 2014). Além da possibilidade de impactos físicos, o trabalho precoce também tem acarretado prejuízos psicossociais como: imagem negativa de si, baixa autoestima, adultização precoce, déficit e evasão escolar e socialização desviante (Alberto, 2007; Alberto, Sousa, Silva, Dantas, Silva, \& Leite, 2010; Lacorte, 2012; Paganini, 2014).

Para este artigo, considera-se acidente de trabalho todo evento proveniente da atividade laboral, seja o acidente típico (aquele que tem presente características de imprevisto e perceptível), seja de trajeto ou de doenças relacionadas ao trabalho (Tortorello, 2014). Em estudo realizado em Ribeirão Preto, $11,1 \%$ dos adolescentes entrevistados relataram terem sido vítimas de acidentes de trabalho, sendo as partes do corpo mais atingidas as pernas e pés. O tipo de agravo mais frequente foi de escoriações e lesões (Pimenta, Freitas, Mendes, Navarro \& Robazzi, 2013).

O trabalho precoce vem sendo reconhecido gradativamente como um problema de saúde pública. Como forma de enfrentar as consequências citadas anteriormente, o Brasil, através do Ministério da Saúde, vem recomendando estratégias para o enfrentamento ao trabalho precoce em três níveis: prevenção, assistência e vigilância em saúde (Ministério da Saúde, 2005). A prevenção ao trabalho precoce é planejada com capacitações, atividades de educação em saúde e mobilizações intersetoriais, para sensibilizar a população e potencializar a participação social para a solução do problema. Essas capacitações e atividades são realizadas por meio do campo da Saúde do Trabalhador, através do Centro de Referência em Saúde do Trabalhador (CEREST) (Ministério da Saúde, 2005; Lacorte, 2012). A assistência promovida pela saúde pública é preconizada pela 
identificação de situação de trabalho, avaliação e diagnóstico, encaminhamentos (Ministério da Saúde, 2005). Todo serviço de saúde pública que preste atenção a crianças e adolescentes, seja atenção básica, de média e alta complexidade, ou de urgência e emergência, deve identificar casos de acidentes e doenças relacionadas ao trabalho, acolhê-los e notificar ao Sistema de Informação de Agravos e Notificação (SINAN) (Santos, 2013). Quanto à vigilância em saúde, ações são executadas e financiadas por todos os entes federativos (União, estado, município e Distrito Federal). Na efetivação das ações, o enfrentamento ao trabalho precoce ocorre primeiramente através dos Distritos Sanitários com monitoramento da saúde de crianças e adolescentes trabalhadores, organização de sistemas de informações e de notificação de casos de trabalho precoce e acidentes de trabalho com crianças e adolescentes (OIT, 2007).

O campo da Saúde do Trabalhador é constituído pela Política Nacional de Saúde do Trabalhador (PNST), que criou como estratégia em 2003 para sua implementação a Rede Nacional de Atenção Integral à Saúde do Trabalhador (RENAST), composta por Centros de Referência em Saúde do Trabalhador (CEREST's). O CEREST tem como atribuições: promover suporte técnico às ações de assistência e vigilância da saúde do trabalhador; recolher, sistematizar e difundir informações, que contribuam com a vigilância; e facilitar os processos de capacitações para profissionais e técnicos do SUS. No que se refere especificamente ao trabalho precoce, O CEREST tem como competência contribuir com a identificação e avaliação da saúde de crianças e adolescentes em situação de trabalho, assim como promover articulação com outros setores do governo e da sociedade na prevenção do trabalho precoce, organizando ações intra e intersetoriais (Pepe, Hoefel, Ximenes, \& Batista, 2009; Santos, 2013).

Em 2004, diante das dificuldades detectadas pelo RENAST, foi publicada a Portaria 777 do Ministério da Saúde, que normatiza a notificação compulsória de agravos à saúde do trabalhador, inclusive de acidentes de trabalho com crianças e adolescentes, e seguindo o fluxo determinado pelo Sistema de Informação de Agravos e Notificação (SINAN) (Ministério da Saúde, 2004; Guedes, 2009). A Portaria 777/MS tem como objetivo integrar a rede de serviços do SUS voltados à assistência ao trabalhador e à vigilância dos ambientes laborais, evidenciando que os acidentes de trabalho merecem atenção especial das políticas públicas do país (Ministério da Saúde, 2005; Santos, Mauro, Brito e Machado, 2009).

Diante da magnitude dos agravos que os acidentes de trabalho causam nos trabalhadores precoces, percebeu-se a necessidade de delinear como questão de estudo as características dos acidentes de trabalho ocorridos com menores de 18 anos, descritas nas fichas de investigação de acidente de trabalho grave do SINAN. Considerando-se tais aspectos, este artigo tem como objetivo caracterizar os acidentes de trabalho ocorridos com crianças e adolescentes, atendidos na rede pública de saúde do município de João Pessoa, descritos em fichas de investigação de acidente de trabalho grave no período de 2011 a 2013, 
além de identificar os principais aspectos para a compreensão do acidente de trabalho, como: as causas, a parte do corpo atingida, o diagnóstico e qual atividade era exercida no momento do acidente.

\section{MÉTODO}

Trata-se de um estudo de caráter quantitativo, corte transversal e descritivo. A partir do método descritivo, procura-se descobrir a frequência com que um fato ocorre, suas características, sua natureza e suas causas, e através deste pode-se classificar, explicar e interpretar os fatos observados (Prodanov \& Freitas, 2013).

Este artigo consiste em um recorte de uma pesquisa maior intitulada "O Enfrentamento ao Trabalho Infantil no Município de João Pessoa. O que há de novo no cenário?", que teve como objetivo analisar o enfrentamento ao trabalho precoce através das políticas de formação profissional, assistência social e saúde. Dentro dessa pesquisa, foi realizado um levantamento dos documentos que estavam relacionados ao trabalho precoce na política pública de saúde. Dentre os dados obtidos, serão utilizados para este artigo os que foram coletados no CEREST, referentes às fichas de investigação de acidentes de trabalho grave.

\section{Amostra}

Encontraram-se 62 Fichas de Investigação de Acidentes de Trabalho Grave de crianças e adolescentes menores de 18 anos que foram atendidos em setores públicos de urgência e emergência do município de João Pessoa. As fichas de acidente de trabalho coletadas para o estudo se referiram ao período de janeiro de 2011 a dezembro de 2013, as quais foram cedidas para consulta pelo CEREST.

As Fichas de Investigação de Acidentes de Trabalho Grave do SINAN possibilitam coletar informações referentes a "Dados Gerais", que informa sobre a fonte notificadora, data do acidente e data da notificação; "Notificação Individual", que traz informações sobre o paciente, como nome, data de nascimento, idade, sexo; "Dados de Residência", contendo informações sobre o endereço do paciente e telefone para contato; "Antecedentes Epidemiológicos", que apresenta a ocupação, o tempo de trabalho na ocupação, local do acidente e dados da empresa contratante, caso haja; "Dados do acidente", contendo informações sobre o horário em que o acidente ocorreu e código do CID-10 da causa do acidente; "Dados do Atendimento Médico", que informa se houve atendimento médico, qual parte do corpo foi atingida, e código do CID-10 sobre o diagnóstico da lesão; "Conclusão", que contém informações sobre a evolução do caso; e, por fim, "espaços para informações complementares e observações".

Registrou-se o número da ficha; ano em que ocorreu o atendimento; sexo, idade e ocupação da criança e adolescente acidentado; quantidade de itens cujos 
motivos são ignorados e de itens sem respostas de cada ficha; código do CID 10 para a causa do acidente; e código do CID 10 do diagnóstico da lesão. As informações das fichas foram transcritas para posteriormente serem tabuladas e analisadas.

\section{Procedimentos}

Em relação aos procedimentos éticos e metodológicos, a princípio, o projeto de pesquisa foi aprovado pelo Comitê de Ética do Centro de Ciências da Saúde da Universidade Federal da Paraíba - CEP/CCS. Logo após, foi encaminhado à Secretaria Municipal de Saúde de João Pessoa para autorização da realização da coleta de dados. Depois de concedido o termo de anuência pela Secretaria, foram feitos agendamentos na Secretaria Municipal de Saúde, nas Vigilâncias Sanitárias Municipais e no CEREST, para levantamento de documentos referentes ao trabalho precoce.

Buscaram-se documentos referentes ao enfrentamento ao trabalho precoce na Secretaria Municipal de Saúde e nas Vigilâncias Sanitárias Municipais, onde não se encontraram registros de ações com essa finalidade; e no CEREST, onde foram encontradas as Fichas de Acidentes de Trabalho Grave com menores de 18 anos, objeto deste estudo, dentre outros documentos em relação ao trabalho precoce: Calendários de reuniões FEPETI/PB (Fórum Estadual de Prevenção e Erradicação do Trabalho Infantil), dados da PNAD sobre exploração do trabalho infantil, diretrizes para atenção integral à saúde de crianças e adolescentes economicamente ativos, por exemplo.

\section{Análise de dados}

Neste estudo, foram analisadas 62 fichas de acidentes que ocorreram com crianças e adolescentes em situação de trabalho no município de João Pessoa, às quais se teve acesso através do CEREST. As informações coletadas nas fichas de acidente de trabalho grave foram categorizadas e transcritas para um banco de dados no software estatístico Statistical Package for the Social Sciences - SPSS, versão 20. E a análise descritiva foi realizada por meio de avaliação de distribuição de frequência das variáveis. A estatística descritiva permite caracterizar os dados da amostra (Ribeiro, 2010).

Para a realização deste estudo, foram considerados como variáveis: ano, sexo, idade, a atividade exercida no momento do acidente, causas dos acidentes, parte do corpo atingida, diagnóstico, quantidade de itens ignorados e quantidade de itens sem respostas. Estas duas últimas categorias foram acrescidas durante a coleta de dados quando se percebeu número considerável de itens que não foram respondidos ou que em suas respostas não traziam informações não especificadas sobre o acidente. Guedes (2009) afirma que essas fichas devem alimentar o SINAN-NET, com transmissão de dados através da rede de computadores. O sistema auxilia a coleta de dados de acidentes e agravos 
relacionados ao trabalho e, por conseguinte, promove a vigilância da saúde do trabalhador, assim como caracteriza os agravos que acometem crianças e adolescentes economicamente ativos, com intuito de colaborar com a erradicação do trabalho precoce.

\section{RESULTADOS E DISCUSSÃO}

Diante dos dados encontrados, apesar de não garantir que todas as crianças e adolescentes que sofreram acidentes de trabalho estão contabilizadas no senso do IBGE, se forem considerados os 62 acidentes de trabalho ocorridos, estes corresponderiam a $1 \%$ das 6.234 crianças e adolescentes em situação de trabalho no município, identificadas pelo IBGE (2013). Este percentual é abaixo da média do Nordeste $(7,1 \%)$ de machucados ou doenças em decorrência do trabalho (IBGE, 2008), mas não deixa de ser significativo diante das metas a serem alcançadas pelo Brasil até 2016: erradicar as piores formas de trabalho precoce; e todas suas formas até 2020 (Comissão Nacional de Erradicação do Trabalho Infantil, 2011).

De acordo com os resultados obtidos através da análise das fichas, se for considerado cada ano, é possível verificar que houve na amostra um aumento do número de acidentes de trabalho ocorridos com menores de 18 anos entre os anos de 2011 e 2013. No ano de 2011, foi observado 30,6\% dos acidentes registrados; em 2012, 32,3\%; e em 2013, 37,1\% (figura 1), totalizando 62 acidentes de trabalho com crianças e adolescentes atendidos pela urgência e emergência da saúde pública e notificados nesse período.

\section{Acidente de Trabalho Infantil por Ano}

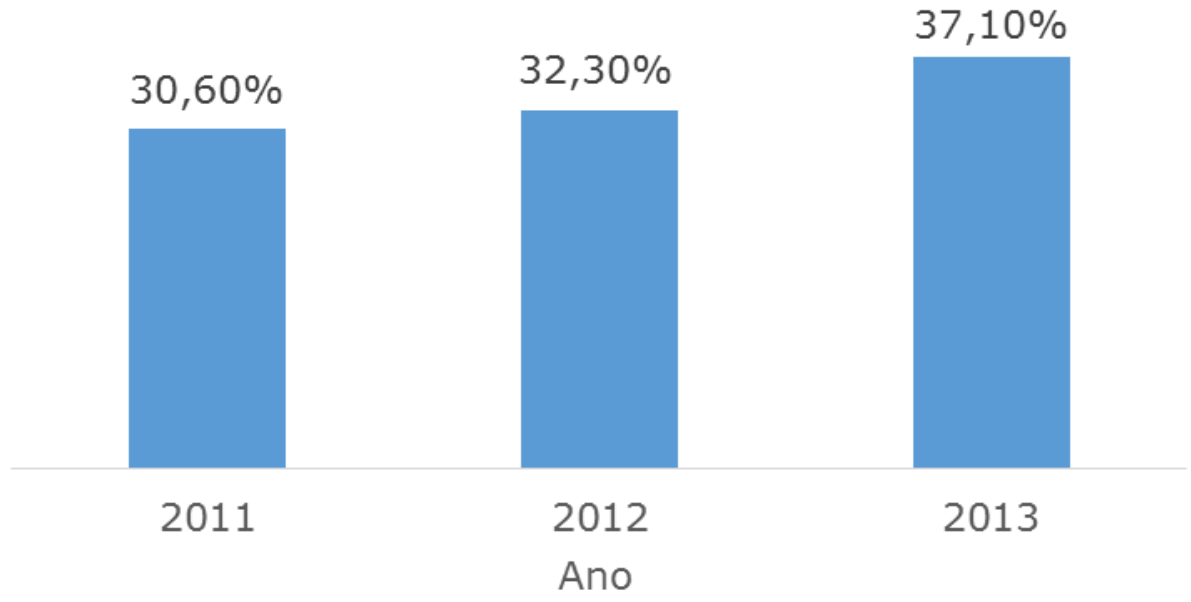

Figura 1. Distribuição de fichas de acidentes de trabalho graves em crianças e adolescentes de 8 a 17 anos, segundo o ano.

Quanto ao sexo das crianças e adolescentes que sofreram acidentes de trabalho, a maioria ocorreu com meninos $(95,7 \%)$, enquanto apenas $4,3 \%$ dos acidentes aconteceram com meninas. Segundo Stropasolas (2012), há uma 
maior inserção de crianças do sexo masculino nas atividades de trabalho precoce, sobretudo ao se tratar de trabalhos considerados mais pesados, como por exemplo indústria, agricultura e construção civil, atividades que requerem contato com ferramentas e equipamentos perigosos.

Como descrito anteriormente, o trabalho precoce é terminantemente proibido pela legislação brasileira até os 14 anos (Presidência da República, 1988/2017). Diante dos dados coletados, 6,5\% dos acidentes notificados ocorreram com crianças de 8 a 13 anos de idade. Os acidentes com adolescentes entre 14 e 15 anos, nos quais só é permitido o trabalho na condição de aprendiz, correspondem a 16,2\%. E, por fim, 77,4\% dos acidentes ocorreram com adolescentes entre 16 e 18 anos incompletos, em que o trabalho é permitido, exceto em condições noturnas, insalubres, penosas ou perigosas (figura 2).

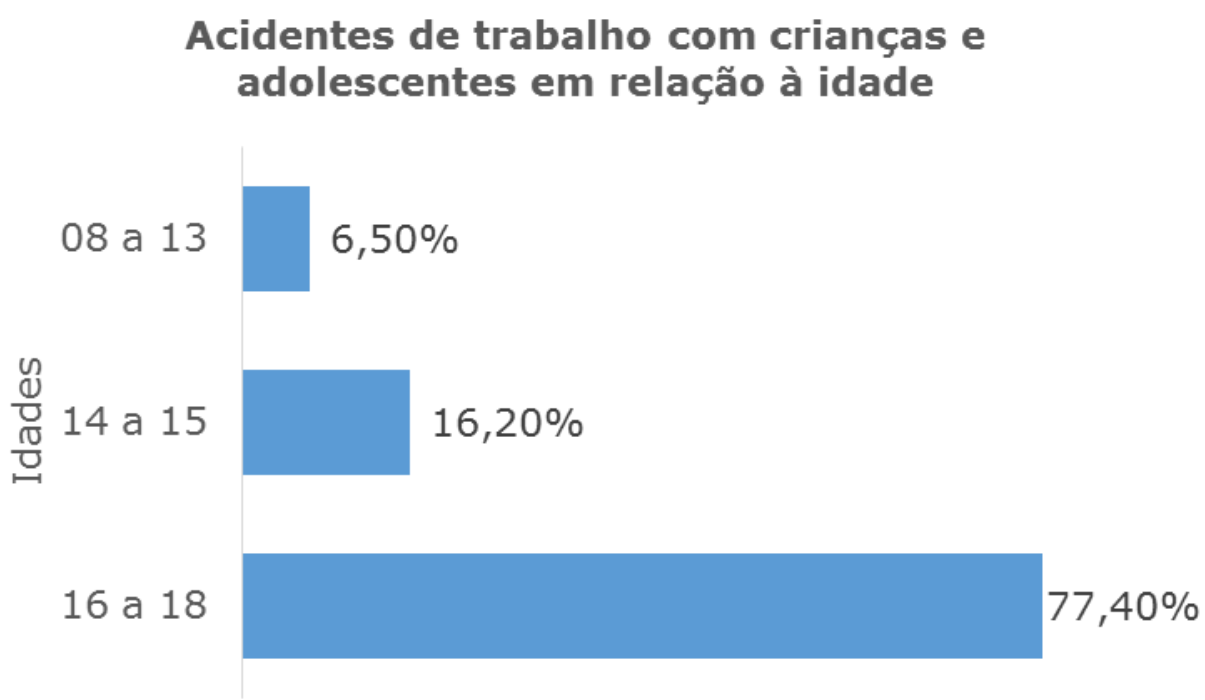

Figura 2. Distribuição de acidentes de trabalho com crianças e adolescentes em relação à idade

De acordo com dados publicados pela OIT (2014), no relatório municipal de João Pessoa, apenas 2,3\% dos adolescentes com idade entre 14 e 15 anos estão em situação legal de trabalho, ou seja, são contratados por empresas como aprendizes, através de programa técnico-profissional. Portanto, diante deste dado, acredita-se que a maioria dos acidentes de trabalho ocorridos com adolescentes de 14 e 15 anos não se refere à condição legal de aprendizes, pois se acidentam em atividades que não são realizadas como forma de aprendizagem técnico-profissional regulamentada.

Em relação aos adolescentes com idades entre 16 e 18 anos, o IBGE (2013) traz que 16\% dos 3.669 adolescentes nessa faixa etária em situação de trabalho estavam empregados com carteira assinada no município. Para caracterizar quais acidentes estudados são resultados de trabalho precoce ilegal é preciso identificar se o tipo de ocupação ou atividade realizada consta na Lista de Piores Formas de Trabalho Infantil. Adolescentes só poderiam exercer os 
trabalhos listados na TIP considerados prejudiciais à saúde e à segurança, caso possuíssem parecer técnico emitido por profissional habilitado em segurança do trabalho atestando a não exposição a riscos que possam comprometer a saúde, a segurança e a moral do adolescente (Presidência da República, 2008).

É importante ressaltar que nas fichas de acidente de trabalho analisadas, vários campos foram preenchidos de forma inadequada, ou seja, estavam em branco ou tinha como resposta que o item foi ignorado. Em média, as fichas tinham 39\% dos 68 itens sem resposta e 4,4\% itens ignorados, que versam principalmente sobre os antecedentes epidemiológicos, dados da residência e dados do acidente. A quantidade considerável de informações ignoradas ou inadequadas dificulta a compreensão de aspectos do acidente.

Os itens que mais ficaram sem resposta foram aqueles referentes aos antecedentes epidemiológicos, que deveriam explicitar os dados sobre a ocupação, empresa contratante e a situação no mercado de trabalho. Acredita-se que essas informações não são descritas nas fichas analisadas, porque na maioria das atividades realizadas por menores de 18 anos não há vínculo legal com empresas (IBGE, 2013), reafirmando o caráter informal ou até mesmo ilegal das atividades realizadas por crianças e adolescentes em situação de trabalho, o que dificulta sua erradicação (Lourenço, 2014).

De acordo com os dados analisados, os acidentes foram causados principalmente por contato com objetos cortantes $(37,1 \%)$, quedas $(21 \%)$ e impacto ou esmagamento com objetos $(19,4 \%)$. Em $8,1 \%$ das fichas, a causa do acidente de trabalho não foi especificada e em uma das fichas este item foi ignorado. Também foram causa de acidentes: acidentes de trânsito $(6,5 \%)$, contato com líquidos quentes $(3,2 \%)$ e penetração de corpos estranhos $(3,2 \%)$. Esses acidentes podem ser explicados por não se considerar a idade ao expor essas crianças e adolescentes a determinadas atividades, que não se adequam à faixa etária; por supervisão inadequada; por contatos com máquinas, substâncias e situações perigosas (Giatti et al., 2014).

Sobre a atividade que as crianças e adolescentes exerciam no momento do acidente de trabalho: $46,4 \%$ estão relacionadas ao comércio e serviços; em $20,8 \%$ das fichas não continha essa descrição; $11,8 \%$ à construção civil; em $9,7 \%$ tinham como ocupação estudante, ou seja, não foi especificada na ficha a atividade realizada no momento do acidente; em $4,8 \%$ das fichas era especificado apenas como ajudante, mas sem especificar o tipo de atividade; $3,2 \%$ à agricultura; e $3,2 \%$ à indústria.

A atividade de trabalho realizada no momento do acidente é um fator que facilita a compreensão do caso ao proporcionar inferências sobre o ambiente de trabalho; possíveis contatos com objetos e máquinas de manuseio arriscado; e exposição a situações de perigo. Preencher esse campo de forma adequada é indispensável para identificar a situação de trabalho precoce. Considera-se também pouco significativo preencher esse campo com termos como "estudante" 
ou "ajudante", os quais dificultam a compreensão do acidente, pois não especifica a atividade realizada. Segundo Alberto (2002), é comum que as atividades realizadas por crianças não sejam consideradas trabalho, mas ajuda a adultos, tornando este tipo de atividade laboral invisível.

Tabela 1. Atividades de trabalho exercidas pelo acidentado

Atividade exercida

Total

Comércio e Serviços

Auxiliar de mecânico

Mecânico

23,2

Lavador de carro

2

3,2

Serralheiro

2

3,2

Garçonete/garçom

23,2

Limpador

1

Auxiliar de soldador

11,6

1,6

Auxiliar de produção

Assistente de vendas

1

1,6

Balconista

1

1,6

Repositor

1

1,6

Investigador

1

1,6

Atendente de lanchonete

1

1,6

Churrasqueiro

1

1,6

Serviços gerais

Auxiliar de administração

1,6

11,6

Doméstica

$1 \quad 1,6$

$1 \quad 1,6$

Vendedor

1

$1 \quad 1,6$

$1 \quad 1,6$

Ajudante de capotaria

$1 \quad 1,6$

Motoboy de lanchonete

$1 \quad 1,6$

Cortador de bolacha

$1 \quad 1,6$

Açougueiro

$1 \quad 1,6$

Ajudante de office-boy

$1 \quad 1,6$

Marceneiro

$1 \quad 1,6$

Sem resposta

$13 \quad 20,8$

Total Comércio e Serviços

42

46,4

Construção Civil

Auxiliar de obras

34,8

Auxiliar de pedreiro

$1 \quad 1,6$

Autônomo em construção

$1 \quad 1,6$




\begin{tabular}{lcc} 
Pedreiro de edifícios & 1 & 1,6 \\
Gesseiro & 1 & 1,6 \\
\hline Total Construção Civil & 7 & 11,8 \\
\hline Agricultura & & \\
Agricultor & 2 & 3,2 \\
\hline Indústria & & \\
Auxiliar de produção & 1 & 1,6 \\
Metalúrgico & 1 & 1,6 \\
Total Indústria & 2 & 3,2 \\
\hline Outros Tipos de Função & & \\
\hline Estudante & 6 & 9,6 \\
Ajudante & 3 & 4,8 \\
Total Outros Tipos de Função & 9 & \\
\hline
\end{tabular}

Nota. $\mathrm{n}=$ total de acidentados.

A maioria dessas atividades se refere ao setor informal de trabalho que, conforme discutido anteriormente, diz respeito à geração de renda por meio de atividades de trabalho desempenhadas pela maioria das pessoas em situação de vulnerabilidade. Este se caracteriza e se diferencia do setor formal, pois consiste em trabalhos que requerem habilidades adquiridas fora do sistema escolar, realizados por pessoas que não são empregadas oficialmente, relações de trabalho mais exploratórias e horários de trabalhos intermitentes e mais prolongados (International Labour Office, 2004). Para Lourenço (2014), o crescimento das relações informais de trabalho, que incluem o trabalho precoce, dificulta as ações fiscalizatórias devido à "não formalização" das atividades, prejudicando o reconhecimento do problema, pois permanece submerso nas relações privadas. O setor informal do trabalho, com atividades mal remuneradas, precárias e desgastantes, constitui-se como um modelo de controle e dominação da infância das classes menos favorecidas, como uma forma de adestramento, não havendo perspectivas de qualificação e de melhoria de vida (Alberto, 2002).

A realização de atividades de construção civil é considerada uma das piores formas de trabalho, da lista TIP (Presidência da República, 2008), relacionada a riscos ocupacionais como, por exemplo, acidentes por queda de nível, acidentes por contato com máquinas, equipamentos e ferramentas. E como repercussão à saúde: fraturas, esmagamentos, traumatismo, contusões, dentre outras. Atividades relacionadas à indústria de transformação também constam na lista, por possíveis riscos como esforços físicos intensos e repetitivos, exposição a altas temperaturas, poeiras inorgânicas e fumos metálicos. E como consequências à saúde, principalmente: DORT/LER (Doenças Osteoarticulares Relacionadas ao Trabalho e Lesão por Esforço Repetitivo) e doenças respiratórias (Presidência da República, 2008). 
Em relação às características do acidente, a maioria teve como parte do corpo atingida os membros superiores, em $56,5 \%$ dos casos; membros inferiores em 17,7\%; e a cabeça/face foi atingida em $8,1 \%$ dos acidentes. Em 11,3\% dos acidentes, a parte atingida não foi especificada. E em 6,5\%, não havia resposta no item. Na pesquisa realizada por Santos et al. (2009), também encontraram resultados em que a parte do corpo mais afetada foram membros superiores: mãos e dedos $(40,4 \%)$, braços antebraços e cotovelo $(19,1 \%)$, seguida por membros inferiores $(20,6 \%)$. Resultados diferentes foram encontrados por Pimenta et al. (2013), nos quais os adolescentes afirmaram que em acidentes de trabalho a parte do corpo mais atingida foram pés e pernas.

Sobre o diagnóstico, os acidentes causaram em sua maioria ferimentos $(27,4 \%)$; contusão $(22,6 \%)$; traumatismo $(11,3 \%) ;$ fratura $(4,8 \%)$; queimadura, amputação, entorse/distensão (3,2\% cada); edema e lesão $(1,6 \%)$. Neste item, em 11,3\% não era especificado o diagnóstico e 6,5\% não tinham resposta.

As prováveis repercussões à saúde relacionadas ao trabalho perigoso estão elencadas na Lista das Piores Formas de Trabalho Infantil (Presidência da República, 2008), são previstas desde afecções músculo-esqueléticas até mutilações, provocando incapacidades temporárias ou permanentes. A literatura afirma que crianças e adolescentes estão mais expostos a riscos, lesões e deformidades físicas causadas em ambiente de trabalho por não terem a condição motora plenamente desenvolvida (Lacorte, 2012; Giatti et al., 2014).

Os resultados obtidos neste estudo alertam para estatísticas relacionadas à assistência e vigilância em saúde somente quando os acidentes de trabalho já aconteceram, ou seja, não houve prevenção nem erradicação do trabalho precoce, pois ele continua acontecendo. Dessa forma, já está instaurada a violação de direitos de crianças e adolescentes em situação de trabalho que acarretam prejuízos à sua saúde. Neste sentido, a caracterização desses agravos é uma forma de auxiliar o monitoramento do impacto do trabalho precoce sobre a saúde, podendo contribuir com a tomada de decisão e ações a nível de assistência e vigilância em saúde, além de reforçar o papel das instituições de saúde com olhar diferenciado na atenção de crianças e adolescentes (Ministério da Saúde, 2004; Ministério da Saúde, 2005; OIT, 2007; Pepe et al., 2009; Santos, 2013). No entanto, necessita-se também de um olhar atento para consequências psicossociais negativas relacionadas ao trabalho precoce (Alberto, 2007; Alberto et al., 2010; Lacorte, 2012; Paganini, 2014), que trazem agravos psicológicos, além de físicos. Na luta pela erradicação do trabalho precoce, é importante considerar a ação das políticas públicas de saúde focadas para a prevenção do trabalho precoce. 


\section{CONSIDERAÇÕES FINAIS}

Buscando delinear como questão de estudo as características dos acidentes de trabalho ocorridos com menores de 18 anos, buscou-se caracterizar os acidentes de trabalho ocorridos com crianças e adolescentes, atendidos na rede pública de saúde, descritos em fichas de investigação de acidente de trabalho grave, além de identificar os principais aspectos para a compreensão do acidente de trabalho, como: as causas, a parte do corpo atingida, o diagnóstico e qual atividade era exercida no momento do acidente.

Diante disso, observou-se que quando se põe em pauta todas as consequências, sejam elas sociais, físicas e/ou psíquicas do trabalho precoce, torna-se evidente o motivo pelo qual é considerado um problema de saúde pública. Para que a realidade do trabalho precoce seja transformada, reafirma-se a necessidade de um trabalho conjunto, entre união, estado, município e sociedade na execução de políticas públicas nos diversos setores, um olhar diferenciado voltado para direitos de crianças e adolescentes e para a erradicação do trabalho precoce.

Os resultados encontrados revelam que crianças e adolescentes em situação de trabalho precoce estão frequentemente expostos a riscos, pois, apesar de haver inserção regulada de adolescentes no mercado de trabalho como aprendizes, muitas vezes as atividades de trabalho não se constituem dessa forma nem seguem as orientações legais. Ao encontrarmos números expressivos sobre acidentes de trabalho envolvendo crianças e adolescentes, deve-se reconhecer a necessidade de preparo dos profissionais da saúde em diversos setores para identificar os riscos e consequências à saúde das pessoas em situação de trabalho. É essencial ainda reafirmar a relevância de uma atuação de políticas públicas de saúde em que seja priorizado a prevenção e erradicação do trabalho precoce. A atenção básica de saúde tem um papel de extremo valor na identificação e notificação de casos de trabalho precoce. Ressalta-se a importância da atuação em todos os níveis de gestão em relação ao combate e erradicação do trabalho precoce, como uma forma de promover ações conscientes do intuito de promover cuidado e proteção de crianças e adolescentes trabalhadores, identificando e notificando o trabalho precoce de forma a se evitar situações de trabalho e acidentes provocados por estas.

Esse estudo apresenta limitações devido ao número restrito de fichas e curto período desse registro no SINAN (apenas três anos - 2011 a 2013), além do mau preenchimento das mesmas. No entanto, trouxe bastante contribuições na discussão acerca do enfrentamento ao trabalho precoce e da vigilância em saúde, justamente por tornar visível o erro no preenchimento das fichas e a ocorrência de acidentes de trabalho com crianças e adolescentes. A partir dessa pesquisa, é possível avançar nessa discussão, buscando a continuidade da análise das fichas em períodos posteriores. 


\section{DECLARAÇÃO DE CONFLITOS DE INTERESSE}

Não há conflitos de interesse.

\section{REFERÊNCIAS}

Alberto, M. F. P. (2002). A dimensão subjetiva do trabalho precoce de meninos e meninas em condição de rua em João Pessoa (PB). (Tese de Doutorado). Universidade Federal de Pernambuco, Recife.

Alberto, M. F. P. (2007). As implicações psicossociais do trabalho precoce em meninos e meninas em condição de rua. Revista Laboreal, 3(2), 9-21. Recuperado em 30 de abril de 2019, de http://laboreal.up.pt/files/articles/2007 12/pt/8 21pt.pdf.

Alberto, M. F. P., Sousa, G. P., Silva, R. M. P., Sousa, O. M. C. G., Dantas, A. P. A., Silva, A. C. S., \& Leite, F. M. (2010). Mapeamento do trabalho Infantil na Paraíba: Um contexto de desenvolvimento para crianças e adolescentes pobres. In V. L. Luna, \& Z. A. Nascimento. (Eds.), Desafios da Psicologia Contemporânea. João Pessoa, PB: Editora Universitária da UFPB.

Comissão Nacional de Erradicação do Trabalho Infantil (2011). Plano Nacional de Prevenção e Erradicação do Trabalho Infantil e Proteção ao Adolescente Trabalhador (2a ed.). Brasília, DF: Ministério do Trabalho e Emprego.

Giatti, L., Campos, M. O., Crespo, C. D., Andrade, S. S. C. A., Barreto, S. M. (2014). Trabalho precoce, marcador de vulnerabilidades para saúde em escolares brasileiros: Pesquisa Nacional de Saúde do Escolar (PeNSE 2012). Revista Brasileira de Epidemiologia, 17-30. http://dx.doi.org/10.1590/18094503201400050003.

Guedes, M. H. M. (2009). Boas práticas nas ações de vigilância na saúde: sobre o Sistema de Informação Nacional de Agravos de Notificação (SINAN-NET). In: S. L. Barker (Eds.). Boas práticas do setor de saúde para a erradicação do trabalho infantil. Brasília: OIT.

Instituro Brasileiro de Geografia e Estatística (2013). Censo Demográfico 2010. Resultados do universo. Recuperado em 23 agosto, 2014, de http://censo2010.ibge.gov.br/trabalhoinfantil/

Instituto Brasileiro de Geografia e Estatística (2008). Aspectos complementares de educação, afazeres domésticos e trabalho - 2006. Rio de Janeiro, RJ: Autor.

International Labour Office. (2004). Child labour: A text book for university students. Geneva, $\mathrm{CH}$ : Author.

Lacorte, L. E. C. (2012). A construção de Políticas Públicas em rede Intersetorial para a Erradicação do Trabalho Infantil em Limeira-SP. (Dissertação de Mestrado). Universidade de São Paulo, São Paulo. 
Lourenço, E. A. S. (2014). Reestruturação produtiva, trabalho informal e a invisibilidade social do trabalho de crianças e adolescentes. Serviço Social \& Sociedade, (118), 294- 317. http://dx.doi.org/10.1590/S010166282014000200005.

Ministério da Saúde. (2004). Portaria no 777, de 28 de abril de 2004. Dispõe sobre os procedimentos técnicos para notificação compulsória de agravos a saúde do trabalhador em rede de serviços sentinela específica. Brasília, DF: Autor.

Ministério da Saúde. (2005). Trabalho infantil: diretrizes para atenção integral à saúde de crianças e adolescentes economicamente ativos. Ministério da Saúde, Secretaria de Atenção à Saúde, Departamento de Ações Programáticas Estratégicas. Brasília, DF: Autor.

Organização Internacional do Trabalho (2007). Módulos de auto-aprendizagem sobre saúde e segurança no trabalho infantil e juvenil. Brasília, DF: Autor.

Organização Internacional do Trabalho (2014). Boletim de dados municipais, município: João Pessoa. Sistema de Indicadores Municipais de Trabalho Decente (SIMTD). Brasília, DF: Autor. Recuperado em 23 agosto, 2014, de http://www.bsb.ilo.org/simtd/download/250750.

Presidência da República (2008). Decreto no 6.481, de 12 de junho de 2008. Regulamenta os artigos 3o, alínea " $d$ ", e 4o da Convenção 182 da Organização Internacional do Trabalho (OIT) que trata da proibição das piores formas de trabalho infantil e ação imediata para sua eliminação. Brasília, DF: Autor.

Presidência da República. (2017). Constituição da República Federativa do Brasil 1988. [Coleção Saraiva de Legislação]. (54a ed.). São Paulo, SP: Saraiva. Original publicado em 1988.

Paganini, J. (2014). Os impactos do Trabalho Infantil para a Saúde da Criança e do Adolescente. VII Mostra de Trabalhos Jurídicos Científicos, (11). Santa Cruz do Sul, RS: EDIUnisc.

Pepe, C. C. A., Hoefel, M. G. L., Ximenes, L., \& Batista, R. A. (2009). A configuração da rede de Atenção Integral à Saúde do Trabalhador do Ministério da Saúde e a Política Nacional de Saúde para a Erradicação do Trabalho Infantil. In S. L. Barker (Ed.), Boas práticas do setor da saúde para erradicação do trabalho infantil. Brasília: OIT.

Pimenta, A. A., Freitas, F. C. T., Mendes, A. M. O. C., Navarro, V. L., \& Robazzi, M. L. C. C. (2013). Acidentes de trabalho ocorridos entre adolescentes. Texto \& Contexto - Enfermagem, 22(2), 279-284. http://dx.doi.org/10.1590/S0104-07072013000200002.

Prodanov, C. C., \& Freitas, E. C. (2013). Metodologia do Trabalho Científico: Métodos e Técnicas de Pesquisa e do Trabalho Científico (2a ed.). Novo Hamburgo, RS: Universidade Feevale. 
Ribeiro, J. L. P. (2010). A investigação e avaliação em psicologia e saúde (2a ed). Lisboa, Placebo Editora.

Santos, M. E. A., Mauro, M. Y. C., Brito, C. G., \& Machado, D. C. (2009). Trabalho precoce e acidentes ocupacionais na adolescência. Escola Anna Nery, 13(4), 824-832. http://dx.doi.org/10.1590/S141481452009000400019.

Santos, S. A. (2013). Política Nacional de Saúde para a Erradicação do Trabalho Infantil e Proteção ao Adolescente Trabalhador: O desafio de construir a atenção integral à saúde de crianças e adolescentes trabalhadores no Sistema Único de Saúde - SUS. Boletim Epidemiológico Paulista, 10(114), 5-16. Recuperado em 30 de abril de 2019, de http://periodicos.ses.sp.bvs.br/scielo.php?script=sci arttext\&pid=S1806$42722013000600002 \&$ lng $=p t \& t$ tng $=p t$.

Stropasolas, V. L. (2012). Trabalho infantil no campo: Do problema social ao objeto sociológico. Revista Latino-americana de Estudos do Trabalho, $17(27), 249-286$.

Tortorello, J. M. (2014). Acidentes de Trabalho. São Paulo, SP: Baraúna.

Sobre as autoras

Lorraine Lacerda Leite é psicóloga pela Universidade Federal da Paraíba. lorrainelale@gmail.com

Gabriela Fernandes Rocha é Doutora em Psicologia Social pela Universidade Federal da Paraíba. gabifernandesrocha@gmail.com

Maria de Fátima Pereira Alberto é Doutora em Sociologia pela Universidade Federal de Pernambuco. Professora Associada III da Universidade Federal da Paraíba. Coordenadora do Núcleo de Pesquisas e Estudos sobre Desenvolvimento da Infância e Adolescência. Pesquisadora Bolsista CNPq nível 2. jfalberto@uol.com.br 
Certificamos que todos os autores participaram suficientemente do trabalho para tornar pública sua responsabilidade pelo conteúdo. A contribuição de cada autor pode ser atribuída como se segue: Gabriela Fernandes Rocha, Lorraine Lacerda Leite e Maria de Fátima Pereira Alberto contribuíram para a conceitualização, investigação e visualização do artigo; Maria de Fátima Pereira Alberto e Lorraine Lacerda fizeram a redação inicial do artigo e Gabriela Fernandes Rocha e Lorraine Lacerda Leite são os responsáveis pela redação final (revisão e edição).

Os autores agradecem ao CEREST (Centro de Referência em Saúde do Trabalhador) pela disponibilidade das fichas de acidente de trabalho. Agradecem o financiamento para pesquisa do Edital Universal do CNPQ; Bolsa Produtividade CNPQ para Maria de Fátima Pereira Alberto; Bolsa CAPES de Doutorado para Gabriela Fernandes Rocha. Agradecem, ademais, a Ana Alayde Saldanha pela leitura de versão inicial do texto.

Recebido em: 31/07/2017

Revisado em: 25/10/2017

Aceito em: 18/01/2018 